\title{
Demonstration of Need of Delegation with Newton's Third Law of Action and Reaction
}

\author{
Rodrigo Fernando Herrera, Felipe Cristóbal Muñoz \\ School of Civil Engineering, Pontificia Universidad Católica de Valparaíso, Valparaíso, Chile \\ Email: rodrigo.herrera@pucv.cl
}

Received 25 March 2016; accepted 17 April 2016; published 20 April 2016

Copyright (C) 2016 by authors and Scientific Research Publishing Inc.

This work is licensed under the Creative Commons Attribution International License (CC BY). http://creativecommons.org/licenses/by/4.0/

(c) (i) Open Access

\begin{abstract}
The article's aims are to present the action of delegation as a need to every business, especially when they get into a process of growth or expansion of their services and the second object is to propose a delegation model. To achieve the first object, a comparison is performed between the need of delegation in a business and Newton's third law of action and reaction, considering as "action" the applicant work force from the clients and as "reaction" the business' capacity to perform a determined work. On the other hand, the proposed "LWPO" model is based in leadership, work, people and objectives of the organization, so it can achieve effectively its strategic. The main feature about the "LWPO" model is that you can select and train contributors in which you may delegate in the future.
\end{abstract}

\section{Keywords}

Delegation, Leadership, Newton's Third Law, LWPO Model

\section{Introduction}

Delegation is the act of putting the fulfillment of tasks in other people that until the present where it is played by one person or a small group of people, corresponding to the high positions of an organization [1]. Delegation is related with giving in to less hierarchical ranks in the performance of unique and relevant work for the organization [2].

It must be clear that the organizational structure of every business implies the assignment of specific chores in specific workstations and is completed by specific people. If you delegate that you can break those "solid" barriers to transfer important (time and transcendence) chores from the high commands to other employees, but also thinking about the organizational structure, in a way that you can give a more relevant role to the contributors, put off overload to the high ranks and allow the expansion of the company [3].

How to cite this paper: Herrera, R.F. and Muñoz, F.C. (2016) Demonstration of Need of Delegation with Newton's Third Law of Action and Reaction. Open Journal of Business and Management, 4, 251-257. 
A lot of medium organizations or companies that are in the search for expansion fail because they are not capable of keeping their service standards when tasks multiply and high ranks do not absorb all the authority and control above the activities performed in the organization [4]. The previous said leaves in evidence the lack of delegation from the company's managers to their middle managers, because of the ignorance about how apply it and in some case the resistance to delegate from the bosses [5].

It is because of that motive that this article is trying to prove that delegation is a necessity in every organization that is looking for expansion or increasing its capacity of reaching jobs or projects. To perform this demonstration, it seeks to make an analogy with Newton's Third Law of action and reaction, namely, "when one body exerts a force on a second body, the second body simultaneously exerts a force equal in magnitude and opposite in direction on the first body". This model will let us analyze the business and the amount of work that is made as opposite forces, where the increase of the force implies job requests to the company. The business will have to be balanced with a rise in the amount of services provided; every time that the company will want to grow and not to become stagnant or die.

\section{Body}

Confirm Newton's Third Law says that when a body “A" exerts a "Fab" force on a body "B", this one exerts a "Fba" force equal in magnitude and opposite in direction on the body "A". It is relevant to highlight that generally bodies A and B have different masses, reason why their accelerations will have different magnitudes.

The applicability of this Law is possible to be found in various everyday situations. In the economic sphere an increase of the amount of products implies a rise in the amount of resources to pay for it, in a construction an increase in the load to be lifted by a crane will cause a change to a higher power machinery. All of these situations will provoke an urgent response of reaction before the increase or decrease of any applicant event.

A company can be considered a network of people with certain hierarchy and a certain number of associated chores to the charge and every team member function [6]. It is true that authority decreases when low in hierarchy [7], as shown in Figure 1.

To link any company operation with Newton's Third Law is necessary to validate these concepts: Assignments asked to the company by customers equal applicant force to a body, and ability to do work by company equal resisting force that a body responds with to the applicant force (Figure 2).

In a medium or small company the people network and its organization work pretty well because their job request equal the ability to receive work from the organization. It is because in this type of company the chain of command has just a few deepening levels, generating that the authority exercised by high commands comes easily to the lowest commands, conforming very hierarchical, authoritarian organizations [8].

When a company is trying to expand or increase work capacity, namely, increase the solicitation of work expected, by Newton's Third Law this would imply that should generate an increase in the bearing capacity (company's work team) equivalent to the expected increase solicitation. This does not always happen, generating several possible scenarios.
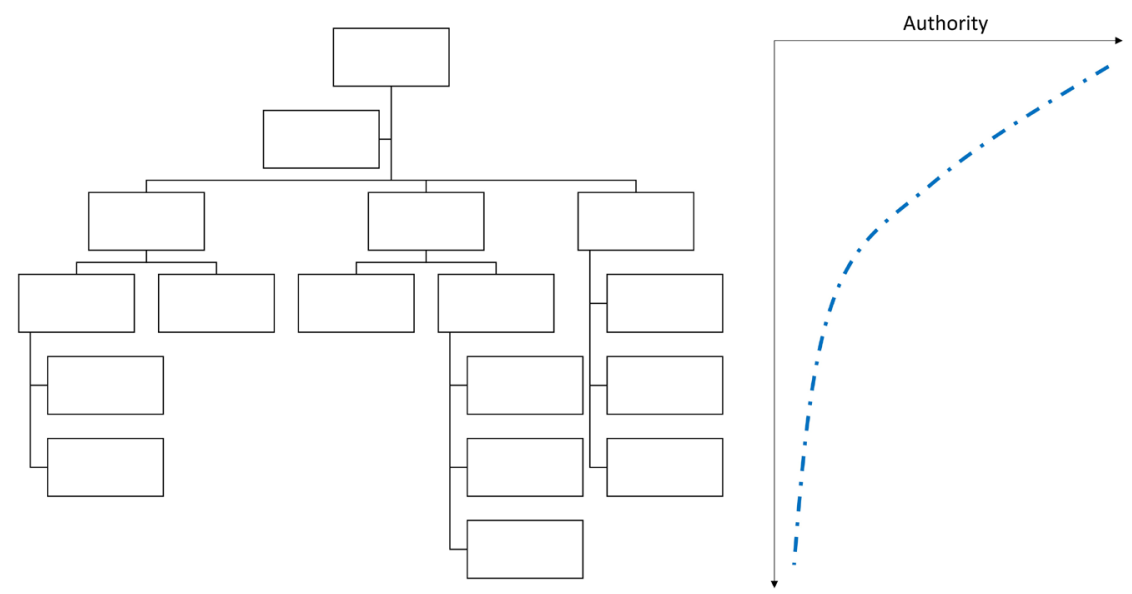

Figure 1. Decrease of authority in an organization. 


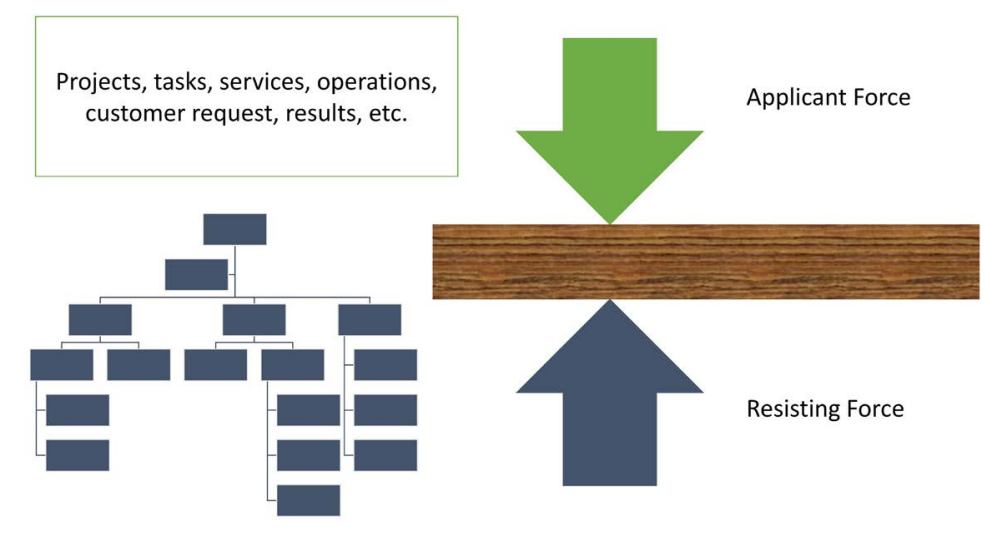

Figure 2. Equivalence organization-Newton's Third Law.

\subsection{Case I: No Work Network Increase}

If the company increases its projects, chores, services, operations, and so on, unplanned, namely, an applicant force increase, without generating an increase strengthening of the work network, namely, keeping without changes the resisting force. It stands to reason that if the applicant force is higher than the resisting force, it won't be generated the waited balance, producing an overexertion of the team reaching failure (Figure 3).

From the company's point of view, what will happen is an overexertion of the existing team, generating a staff fatigue affecting health conditions, occupational safety of human resource and at the same time, the quality of work entrusted, no meeting deadlines and others nonconformities that lead to failure.

\subsection{Case II: Increase the Work Network, But No Delegating Enough Authority in the Medium Commands}

Another scenario that would lead to failure of the organization anyway, is when the organization prevents a greater work effort (applicant force) and therefore increases the network work trying to equate the applicant with the resisting force and keep the balance in the organization, however it is not enough.

Increased work network is essential and a first step to balance the applicant and resisting forces, but this is not enough to achieve this goal, since not delegating the necessary authority to middle managers, power management fails to permeate into everyone in the organization generating a weakness in the resistant structure and therefore a weakness and subsequent breakup of the organizational structure (Figure 4).

\subsection{Case III: Increase Work Network and Delegate Authority to Middle Managers}

For a company to take the decision to increase its project portfolio, tasks, services, operations, etc., namely, increase the applying effort, it must have strengthened and prepared its organization for it. That is why the increase in balance forces raised should start by increasing resisting forces; namely, prepare a team, tools and machineries to confront applications and to balance forces.

The main column of any organization is the people, reason why company's strengthening must be focused principally in them. The first wrong idea that comes to mind in the staff increase, however nothing will change increasing the staff if the ones that control and direct that staff and the tasks is a limited group (bigger responsibilities and charges are generated just by a few people) and corporate control becomes complex and risky. In this situation, a company is growing and trying to resist the working level rise, but at a high and risky cost for the highest ranks.

In this dramatic situation, the act of 'delegating' comes in response to the possibility of growth and in addition to effective process control. Delegating authorities and sharing responsibilities with the middle managers free the increasing loads to higher authorities, decreasing failure possibilities to the growing mass of tasks and people (Figure 5). On the other side, accuracy and effectiveness increase giving authority to people that know deeply the specialty, the production process or the goal to achieve that is being delegated [1].

The Universal History has shown that the power (decision making) by one or a few people just provoke biases in the political decisions and ways to create, innovate and organize. To delegate to others decisions, tasks and 


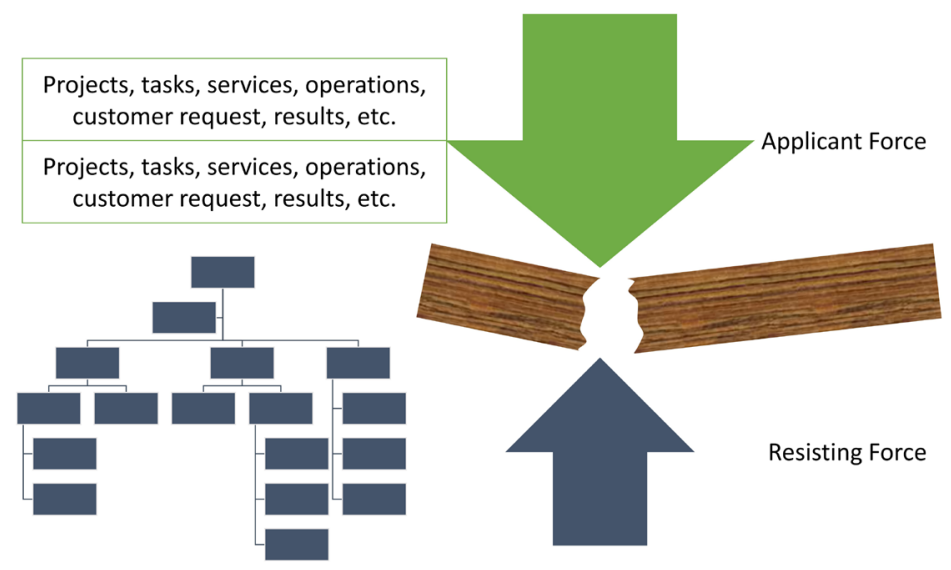

Figure 3. Keep work network.

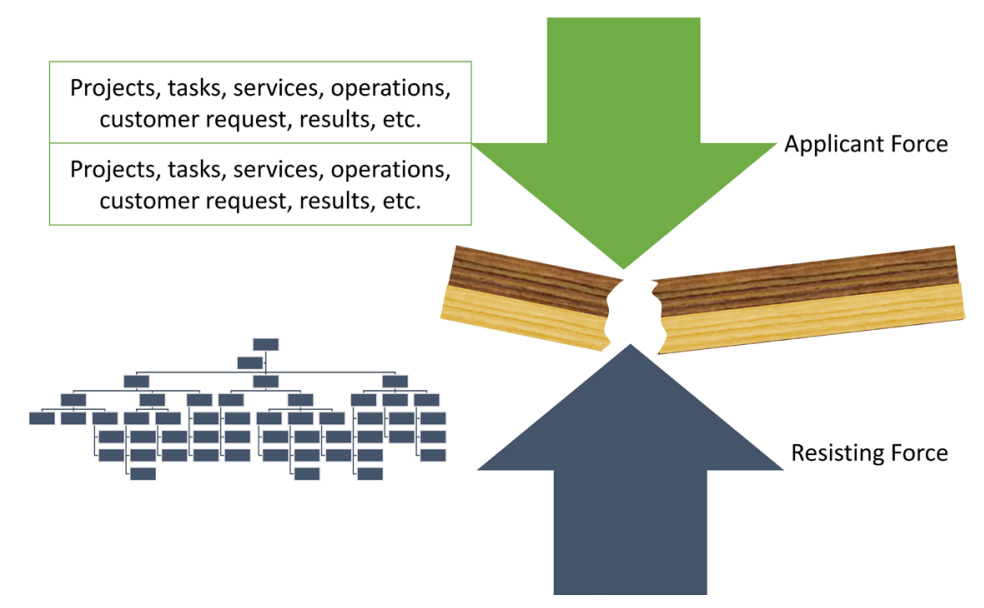

Figure 4. Increase work network and delegate authority to middle managers.

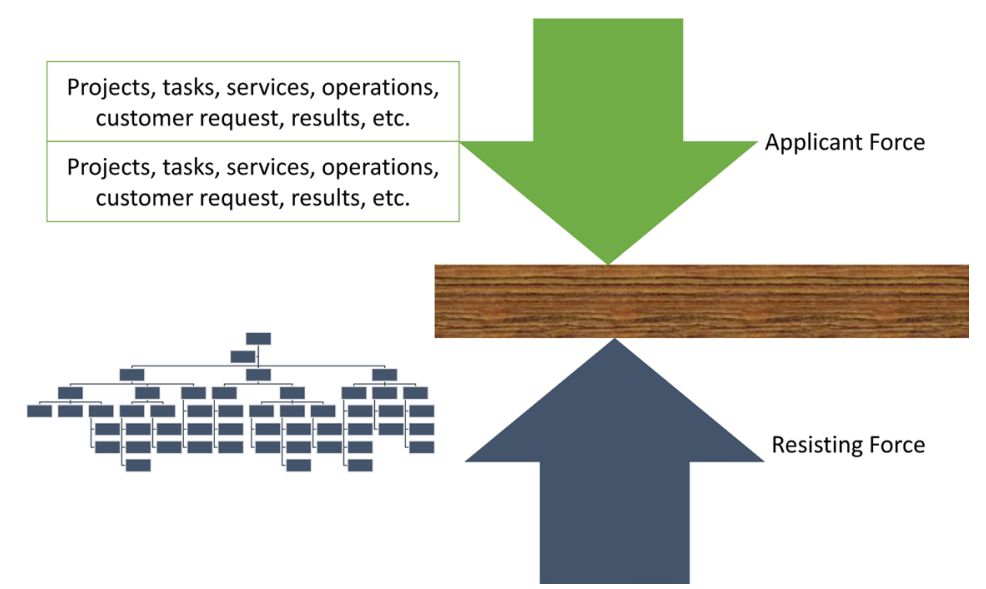

Figure 5. Increase work network and delegate authority.

objectives will refresh processes and departments within the organization [9].

It is already understood the narrow and imperious relation between the company's growth and delegation of tasks, however it means only an initial arrangement for wanting to face successfully growth thereof. It is necessary to implement a method that allows us to link the major variables involved in the company along with the delegation. 
To succeed in this process of delegation (and therefore in business growth) we propose the LWPO model: leadership-work-persons-objectives that gathers the four key features to consider within an organization to achieve the desired balance of forces in the development stages.

The "art" of delegating starts with leadership, the existence of a person (general and ideally high commands) that takes the initial decision. A leader boss (being a boss does not mean being a leader) will be responsible for identifying targets, tasks and necessary people in the process [10]. A boss has clear (or should have) that goals and tasks must be performed to achieve those goals; he knows how to make a big part of the work and so far he plays effectively. On the other side, a leader knows how to be a boss, but also is capable of identifying the right person, either for its skills or knowledge, to make those tasks and achieve goals, but is also capable of delegating in those selected people the achievement of targets (it is not only the execution of proposed tasks), understanding and allowing with that; innovation in processes and routines, always stuck to deadlines and framed in the company values [10]. Figure 6 shows schematically the model LWPO, on top; leader's management within its organization.

The first step required by the LWPO model is to select and train employees which may delegate in the future. Such training profile must be related to the formation of business leaders, thereby generated a virtuous chain inside the organization. To make this right training, researchers Paul Hersey and Ken Blanchard [11], determined in the second half of the XX century the theory of "Situational Leadership" model that provides the basis for actions to be taken by the leader with colleagues according to the level of development or maturity they have reached. The researchers suggest four levels of development and in turn four forms of leadership that are directly related.

Figure 7 shows the relationship between leadership style and behavior of employees. Clearly for the growth of the company and the achievement of the objective of delegation it is necessary that the organization is given the last couple shown, but to achieve this you must go through the entire process (or necessary by level of development).

Achieving recent levels of development will have an ideal and appropriate team that led to company's successful growth. This way people will have been trained capable for receiving objectives and develop methodologies and processes own-account for the performance thereof. Managers should pay attention to the training of empowerment leadership behaviors like providing support, decentralizing and participative decision-making, leading the team by assuming new responsibilities [12].

The formation of suitable persons must be accompanied immediately by the generation of collective responsibility, which seek to consolidate the practices and processes that lead to the fulfillment of the objectives; responsibility is now not only do the job, but is responsible for compliance with the objectives (results). This effectively raises the model of leadership with accountability, current that, as indicated in Figure 8, complete with the difference between work and results, matching both terms on the occasion of achieving the desired and beneficial delegation of responsibility.

\section{Conclusions}

According to the evidence presented can be drawn the following conclusions and recommendations.

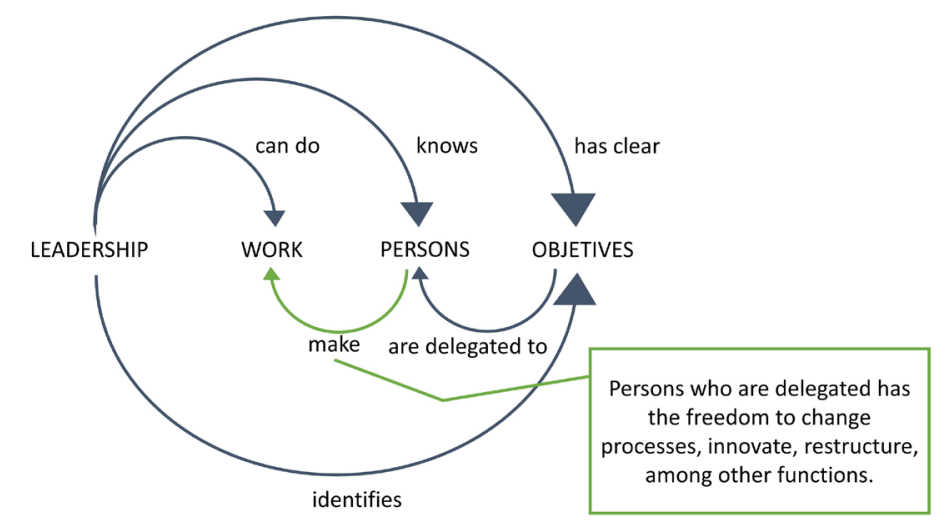

Figure 6. LWPO model. 


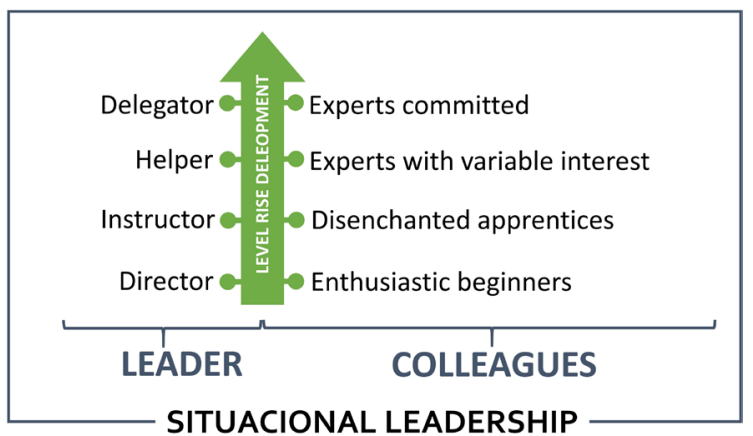

Figure 7. Situational Leadership.

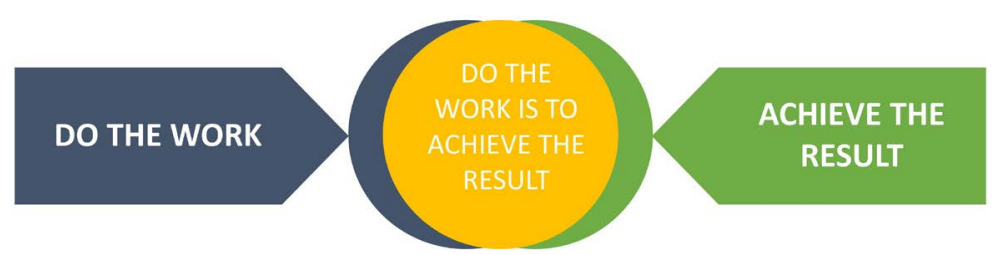

Figure 8. Model of Leadership whit accountability.

Any company that wants to expand its portfolio of projects or expand their tasks and operations should at least expect an increase and strengthening of its organizational structure. This does not mean simply increasing staff shall be sufficient to sustain the charge of requesting new work conditions.

To strengthen the organizational structure, it will be the obligation of company's senior executives to delegate authority to middle managers, so that they in turn are able to empower its tasks, activities and teams. If this does not occur, the company will not be able to have a coherent structure at all levels and therefore will generate weaknesses in the organization that will be the cause of failure before a heavy workload.

Senior managers will be responsible for leading the process of empowering people. For this is proposed the leadership-work-persons-objectives or LWPO model which provides the framework for how bosses must become leaders to be clear about objectives to be fulfilled in a company and to train and select people to delegate the implementation thereof, giving freedom in the form of execution (clearly linked to the own considerations of each particular situation).

\section{References}

[1] Musso, F. (2005) Delegation and Retro Delegation of Directive Function. Teacher Support Documents Series "Development of Contents of Course Programs”.

http://mazinger.sisib.uchile.cl/repositorio/ap/instituto_de_asuntos_publicos/m200510271616dad1.pdf

[2] Ceridian Corporation (2006) Learning to Delegate.

http://people.rice.edu/uploadedFiles/People/TEAMS/Learning\%20to\%20Delegate.pdf

[3] Action Group (2006) Delegating: A Challenge for SMEs. Action Group.

[4] Herrero, E. (2015) What Companies Forget When They Grow up? Forbes: Nothing Personal, Just Business. http://forbes.es/actualizacion/4317/8-cosas-que-las-empresas-olvidan-al-crecer

[5] Melé, D. (2013) The Fear of Delegating: A Question of Absence of Moral Competence? IESE Business School, Universidad de Navarra, Lecture of Business Ethics.

http://blog.iese.edu/eticaempresarial/2013/07/02/el-temor-a-delegar-una-cuestion-de-falta-de-competencias-morales/

[6] De Carlos, C. (2011) Administration and Direction for Business and Social Development. Edtions Universitarias de Valparaíso, Valparaíso.

[7] Project Management Institute (2013) A Guide to the Project Management Body of Knowledge (PMBOK). 5th Edition, Autor.

[8] Cleri, C. (2007) The Book of SMEs. Granica, Buenos Aires.

[9] Robledo, C.P. (2011) Theimportance of Delegating. BDO México. 
[10] Parada, C. (2013) Delegating Empowerment. Toast Masters International, Mission Viejo. http://www.tmdistrito34.org/archivos/educativo/liderazgo/de_excelencia/05-delegar-para-facultar.pdf

[11] Hersey, P. and Blanchard, K.H. (1977) Management of Organizational Behavior 3er Edition-Utilizing Human Resources. Prentice Hall, New Jersey.

[12] Liu, Y.Y. (2015) The Review of Empowerment Leadership. Open Journal of Business and Management, 3, 476-482. http://dx.doi.org/10.4236/ojbm.2015.34049 